\title{
Rhodium/phosphoramidite-catalyzed asymmetric arylation of aldehydes with arylboronic acids $\uparrow$
}

\author{
Richard B. C. Jagt, ${ }^{a}$ Patrick Y. Toullec, ${ }^{a}$ Johannes G. de Vries, ${ }^{* b}$ Ben L. Feringa ${ }^{* a}$ and Adriaan J. Minnaard ${ }^{* a}$ \\ Received 3rd January 2006, Accepted 21st January 2006 \\ First published as an Advance Article on the web 31st January 2006 \\ DOI: 10.1039/b518311a
}

Phosphoramidites are effective chiral ligands in the rhodium catalyzed addition of arylboronic acids to aldehydes providing up to $75 \%$ enantioselectivity and up to $96 \%$ isolated yield.

Enantiopure diarylmethanols are key structural elements in a number of pharmacologically active compounds and therefore represent important synthetic targets. ${ }^{1}$ An attractive route to chiral enantiopure diarylmethanols is the catalytic asymmetric addition of aryl organometallic reagents to aldehydes. ${ }^{2}$ Diphenylzinc has been used as a phenyl transfer reagent, but its background reaction with aldehydes makes it difficult to achieve high stereoselectivity. ${ }^{3}$ This undesired activity could be moderated by the simultaneous use of diethylzinc. ${ }^{4}$ Recently, Bolm et $a l .{ }^{5}$ reported an excellent method in which the aryl transfer reagent was generated by mixing arylboronic acids with an excess of diethylzinc. ${ }^{6}$ Arylboronic acids have received increasing attention as arylating reagents because they are shelf stable, readily available, and compatible with a large variety of functional groups. ${ }^{7}$ From a practical point of view, the development of an efficient protocol for their direct addition to aldehydes would be highly desirable.

The addition of arylboronic acids to aromatic aldehydes was initially reported by Miyaura in 1998 employing rhodium catalysts. ${ }^{8,9}$ With MeO-MOP as the chiral ligand, $41 \%$ ee was obtained for the addition of phenylboronic acid to 1-naphthaldehyde. Subsequent attempts to improve the enantioselectivity of this reaction remained unsuccessful. ${ }^{10}$ The development of suitable chiral ligands for this transformation is still a major goal.

Recently we have demonstrated that rhodium-catalyzed conjugate additions of arylboronic acids to enones can be achieved with high efficiency and excellent enantioselectivities by employing the monodentate phosphoramidite L1 (Fig. 1). ${ }^{11}$ Phosphoramidites
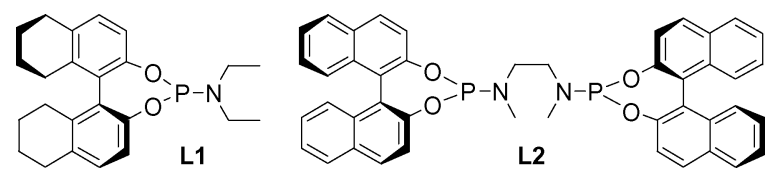

Fig. 1 Chiral monodentate (L1) and bidentate (L2) phosphoramidites.

${ }^{a}$ Department of Organic and Molecular Inorganic Chemistry, Stratingh Institute, University of Groningen, Nijenborgh 4, 9747 AG, Groningen, The Netherlands.E-mail: a.j.minnaard@rug.nl,b.l.feringa@rug.nl

${ }^{b}$ DSM Research, Life Sciences-Advanced Synthesis \& Catalysis, P.O. box 18, 6160 MD, Geleen, The Netherlands

$\dagger$ Electronic supplementary information (ESI) available: Experimental procedures and ${ }^{1} \mathrm{H}$ NMR and ${ }^{13} \mathrm{C}$ NMR spectral data and analytical data for products 3a-i. See DOI: 10.1039/b518311a comprise a low-cost class of ligands that are easily tunable and therefore highly suitable for a combinatorial approach in the development of asymmetric catalysts. ${ }^{12}$ Herein we report the efficient rhodium-catalyzed addition of arylboronic acids to aldehydes with enantioselectivities up to $75 \%$ using bidentate phosphoramidite L2 (Fig. 1).

Preliminary studies were carried out with $p$-chlorobenzaldehyde (1a), 3 equiv. of phenylboronic acid (2a), and a catalyst prepared in situ from $3 \mathrm{~mol}^{\%} \mathrm{Rh}$ (acac)(eth) $)_{2}$ and $7.5 \mathrm{~mol} \%$ phosphoramidite L1. Although a promising $41 \%$ enantioselectivity was obtained, conversion was incomplete under the aqueous conditions commonly used for the rhodium-catalyzed conjugate addition of arylboronic acid (dioxane : $\mathrm{H}_{2} \mathrm{O}=10: 1$, Table 1 , entry 1 ).

Interestingly, it was observed that the reaction also proceeded in non-protic media without water as a co-solvent (entries 2, 4, and 6). In conjugate addition processes of arylboronic acids, water or alcohol additives have been proven essential to protonate the rhodium oxa- $\pi$-allyl intermediate and thus achieve catalytic activity. ${ }^{13}$ If work-up with $12.5 \%$ aqueous ammonia was omitted, a mixture of product $\mathbf{3} \mathbf{a}$ and a considerable amount of a product related compound was found. This intermediate was identified as borate ester 4 (see proposed catalytic cycle in Scheme 1). ${ }^{14}$ The presence of $\mathbf{4}$ suggests that under these conditions the alcoholate functionality of species $\mathbf{D}$ is able to induce the transmetallation

Table 1 Solvent variation in the rhodium-catalyzed asymmetric addition of arylboronic acids to aldehydes ${ }^{a}$

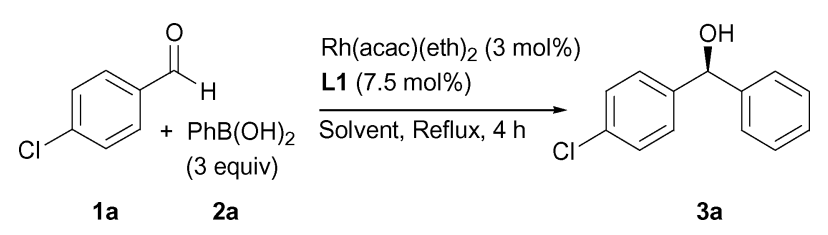

\begin{tabular}{llll}
\hline Entry & Solvent & Conversion $(\%)^{b}$ & ee $(\%)^{c}$ \\
\hline 1 & Dioxane $/ \mathrm{H}_{2} \mathrm{O}(10: 1)$ & 79 & 41 \\
2 & Dry dioxane & 87 & 36 \\
3 & Toluene $/ \mathrm{H}_{2} \mathrm{O}(10: 1)$ & 17 & 44 \\
4 & Dry toluene & 26 & 20 \\
5 & Tetrahydrofuran $/ \mathrm{H}_{2} \mathrm{O}(10: 1)$ & 24 & 44 \\
6 & Dry tetrahydrofuran & 31 & 42 \\
7 & 1,2-Dimethoxyethane $/ \mathrm{H}_{2} \mathrm{O}(10: 1)$ & 45 & 30 \\
8 & 2-Propanol & $>99$ & 52
\end{tabular}

${ }^{a}$ Reactions were carried out on $0.2 \mathrm{mmol}$ scale in $2 \mathrm{~mL}$ of solvent at reflux for $4 \mathrm{~h}$ with 3 equiv. of phenylboronic acid (2a) in the presence of a catalyst generated from $3 \mathrm{~mol} \% \mathrm{Rh}(\mathrm{acac})(\mathrm{eth})_{2}$ and $7.5 \mathrm{~mol} \%$ of phosphoramidite L1. ${ }^{b}$ Conversion determined by ${ }^{1} \mathrm{H}-\mathrm{NMR}$ after workup with $12.5 \%$ aqueous ammonia. ${ }^{c}$ Determined by chiral HPLC. 
Table 2 Scope of the rhodium-catalyzed asymmetric addition of arylboronic acids to aldehydes ${ }^{a}$

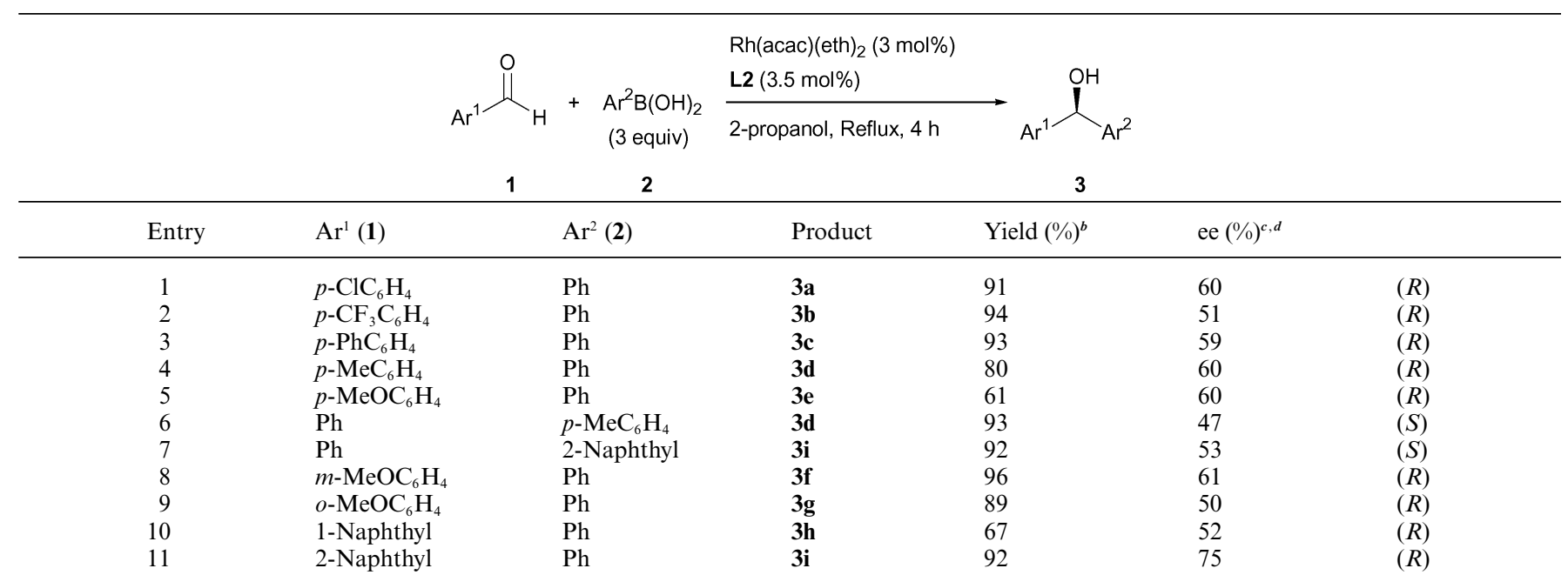

${ }^{a}$ Reactions were carried out on $0.2 \mathrm{mmol}$ scale in $2 \mathrm{~mL}$ of 2-propanol at reflux for $4 \mathrm{~h}$ with 3 equiv of boronic acid 2 in the presence of a catalyst generated from $3 \mathrm{~mol} \% \mathrm{Rh}(\mathrm{acac})(\mathrm{eth})_{2}$ and $3.5 \mathrm{~mol} \%$ of bidentate phosphoramidite $(S, S)$-L2 ${ }^{b}$ Isolated yields after column chromatography on silica gel (heptane:EtOAc $-15: 1$ ). ${ }^{c}$ Determined by chiral HPLC. ${ }^{d}$ The absolute configuration of $\mathbf{3 a}-\mathbf{i}$ was established by comparison of the optical rotation with literature values or deduced by analogy (see electronic supplementary information (ESI) $\dagger$ ).

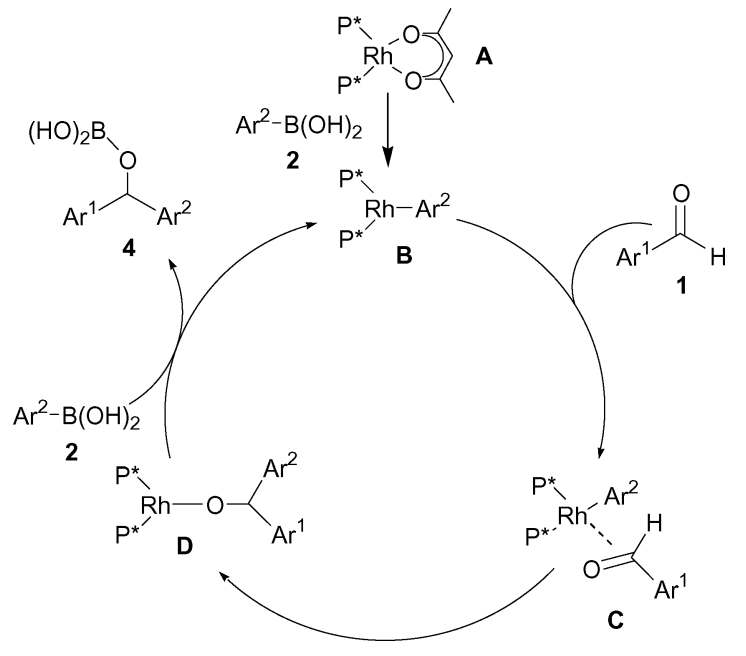

Scheme 1 Proposed mechanism.

step between arylboronic acids and the rhodium center. Due to the equilibrium between phenylboronic acid and its boroxine trimer, the water content can not be defined. Although formation of $\mathbf{4}$ from the product and boronic acid during the reaction can not be excluded, the 3a : $\mathbf{4}$ ratio increased during the course of the reaction, pointing to slow hydrolysis of borate ester 4 .

A study of various solvents showed that 2-propanol is the most suitable solvent for this reaction (compare entries 1-8), increasing both reactivity and enantioselectivity. Full conversion is obtained within $4 \mathrm{~h}$ resulting in $52 \%$ ee. Because of the protic nature of 2-propanol, quenching with aqueous ammonia is not required. Using these optimized conditions, the parallel synthesis and in situ screening of a diverse library of phosphoramidites indicated that bidentate ligand $\mathbf{L} \mathbf{2}$ will provide the highest enantioselectivity in this reaction. Full conversion was reached within $4 \mathrm{~h}$ and product 3a was obtained in $91 \%$ isolated yield with $60 \%$ ee (Table 2, entry 1).

The rhodium-catalyzed arylation of aldehydes is compatible with a wide range of functional groups (Table 2). As already observed by Miyaura ${ }^{8}$ and Frost ${ }^{10}$, the reaction is rather sensitive to electronic effects both in aldehyde and arylboronic acid. Phenylation of aldehydes with electron-withdrawing chloroand trifluoromethyl-substituents at the para position gave the corresponding alcohols $\mathbf{3 a}$ and $\mathbf{3 b}$ in high yields with $60 \%$ and $51 \%$ enantioselectivity, respectively (entries 1 and 2). In the case of a para-phenyl group, the product could be isolated in $93 \%$ yield with $59 \%$ ee (entry 3). Substrates with electron-donating methoxyand methyl-substituents at the para position both provided $60 \%$ ee, but did not proceed to full conversion in $4 \mathrm{~h}$ (entries 4 and 5). The alternative reaction of benzaldehyde with substituted arylboronic acids did give high yields but showed decreased enantioselectivities (entries 6 and 7). A slightly lower ee and reactivity is also observed for sterically hindered substrates like ortho-methoxy benzaldehyde (entry 9) and 1-naphthyl carboxaldehyde (entry 10). On the contrary, 2-naphthyl carboxaldehyde could be phenylated in $92 \%$ isolated yield with an ee of $75 \%$ (entry 11 ).

In summary, catalytic asymmetric synthesis of diarylmethanols has been realized with good enantioselectivities (up to $75 \%$ ) and high isolated yields (up to $96 \%$ ) employing a rhodium/phosphoramidite catalyst system.

\section{Acknowledgements}

Financial support from DSM, the Ministry of Economic Affairs, and the Dutch Science Foundation NWO/CW, administered through the $\mathrm{CW} / \mathrm{Combichem}$ program, is gratefully acknowledged. We thank Mr. E. P. Schudde and Mrs. T. D. TiemersmaWegman for technical support. 


\section{References}

1 Y. Bolshan, C.-Y. Chen, J. R. Chilenski, F. Gosselin, D. J. Mathre, P. D O'Shea, A. Roy and R. D. Tillyer, Org. Lett., 2004, 6, 111; M. Botta, V. Summa, F. Corelli, G. D. Pietro and P. Lombardi, Tetrahedron: Asymmetry, 1996, 7, 1263; S. Stanev, R. Rakovska, N. Berova and G. Snatzke, Tetrahedron: Asymmetry, 1995, 6, 183; C. M. Spencer, D. Foulds and D. H. Peter, Drugs, 1993, 46, 1055; A. F. Casy, A. F. Drake, C. R. Ganellin, A. D. Mercer and C. Upton, Chirality, 1992, 4, 356.

2 For a recent review on metal-catalyzed asymmetric arylation reactions, see: C. Bolm, J. P. Hildebrand, K. Muñiz and N. Hermanns, Angew. Chem., Int. Ed., 2001, 40, 3284.

3 R. Noyori and M. Kitamura, Angew. Chem., Int. Ed. Engl., 1991, 30, 49; K. Soai and S. Niwa, Chem. Rev., 1992, 92, 883; L. Pu and H.-B. Yu, Chem. Rev., 2001, 101, 757; C. Bolm and K. Muñiz, Chem. Commun., 1999, 1295.

4 C. Bolm, N. Hermans, J. P. Hildebrand and K. Muñiz, Angew. Chem., Int. Ed., 2000, 39, 3465; J. Rudolph, C. Bolm and P. O. Norrby, J. Am. Chem. Soc., 2005, 127, 1548.

5 C. Bolm and J. Rudolph, J. Am. Chem. Soc., 2002, 124, 14850.

6 For recent examples, see: J.-X. Ji, J. Wu, T. T.-L. Au-Yeung, C.-W. Yip, R. K. Haynes and A. S. C. Chan, J. Org. Chem., 2005, 70, 1095 and references cited therein.

7 N. Miyaura and A. Suzuki, Chem. Rev., 1995, 95, 2457; Boronic Acids: Preparation and Applications in Organic Synthesis and Medicine, ed. D. G. Hall, Wiley-VCH, Weinheim, 2005.
8 M. Saikai, M. Ueda and N. Miyaura, Angew. Chem., Int. Ed., 1998, 37, 3279.

9 For addition of arylboronic acids leading to racemic aldehydes, see reference 8 and: M. Ueda and N. Miyaura, J. Org. Chem., 2000, 65, 4450; A. Fürstner and H. Krause, Adv. Synth. Catal., 2001, 343, 343.

10 T. Focken, J. Rudolph and C. Bolm, Synthesis, 2005, 429; C. Moreau, C. Hague, A. S. Weller and C. G. Frost, Tetrahedron Lett., 2001, 42, 6957.

11 J.-G. Boiteau, R. Imbos, A. J. Minnaard and B. L. Feringa, Org. Lett., 2003, 5, 681; J.-G. Boiteau, R. Imbos, A. J. Minnaard and B. L. Feringa, Org. Lett., 2003, 5, 1385, also see: J.-G. Boiteau, A. J. Minnaard and B. L. Feringa, J. Org. Chem., 2003, 68, 9481; A. Duursma, R. Hoen, J. Schuppan, R. Hulst, A. J. Minnaard and B. L. Feringa, Org. Lett., 2003, 5, 3111; R. B. C. Jagt, J. G. de Vries, B. L. Feringa and A. J. Minnaard, Org. Lett., 2005, 7, 2433.

12 L. Lefort, J. A. F. Boogers, A. H. M. de Vries and J. G. de Vries, Org. Lett., 2004, 6, 1733; A. Duursma, L. Lefort, J. A. F. Boogers, A. H. M. de Vries, J. G. de Vries, A. J. Minnaard and B. L. Feringa, Org. Biomol. Chem., 2004, 2, 1682.

13 T. Hayashi, M. Takahashi, Y. Takaya and M. Ogasawara, J. Am. Chem. Soc., 2002, 124, 5052.

14 Borate ester 4 has a characteristic benzhydrilic proton signal in ${ }^{1} \mathrm{H}$ NMR at $6.21 \mathrm{ppm}$ and was further identified by negative ion ESIMS, which gave a specific isotope pattern for $\mathrm{C}_{13} \mathrm{H}_{11} \mathrm{ClBO}_{3}{ }^{-} . \mathrm{NH}_{3}(\mathrm{M}-$ $\left.\mathrm{NH}_{4}{ }^{+}\right): m / z 277.1\left(15 \%,{ }^{10} \mathrm{~B},{ }^{35} \mathrm{Cl}\right), 278.1\left(100 \%,{ }^{11} \mathrm{~B},{ }^{35} \mathrm{Cl}\right), 279.0(25 \%$, $\left.{ }^{10} \mathrm{~B},{ }^{37} \mathrm{Cl}\right), 280.0\left(25 \%,{ }^{11} \mathrm{~B},{ }^{37} \mathrm{Cl}\right)$. 